\title{
Teaching System of Integrated Automation of Filling Dosage Wagons for Building Materials
}

\author{
Israel F. Benítez-Pina ${ }^{1}$, Michel Sanz-Perez ${ }^{2}$, Jose Rogelio Martin-Escalona ${ }^{2}$ \\ ${ }^{1}$ Doctor em Ciencias Tecnicas Universidad de Oriente (UO, Cuba). Profesor de Controle e Automacao da Escola Superior de \\ Tecnologia da Universidade do Estado do Amazonas (EST-UEA, AM, Brasil). E-mail: benitez.israel@ gmail.com. \\ ${ }^{2}$ Profesores Dpto. de Controle Automático, Facultad Ing. Eléctrica, Universidad de Oriente. Santiago de Cuba, Cuba. E-mail: \\ msanz@ fie.uo.edu.cu, jrmescalona@ fie.uo.edu.cu
}

\begin{abstract}
Formal design in Petri nets (PN) model of filling wagons in laboratory process is presented with subsequent translation into programmable logic controllers (PLC) language and a monitoring and data acquisition software (SCADA) to the training facility. Use professional tools to model, verify and validate the proposed automation, where two new steps for proper formation of the PN are added, and programming, communication and monitoring of the implementation. With the implementation of this laboratory facility is has a near real automation system, usable in various undergraduate and postgraduate courses tool. The use of formal modeling, OPC communication and supervisory design allow tuning of the application and is an example to follow in teaching of Automation.
\end{abstract}

Keywords: Petri Nets Modeling, OPC communication systems, industrial monitoring, Teaching of Automation.

\section{Sistema de Enseñanza de Automatización Integrada de Llenado de vagones para dosificación de material de construcción}

\section{RESUMEN}

Se presenta el diseño formal en Redes de Petri (PN) de un proceso de llenado de vagones para una maqueta de laboratorio, con su posterior traducción a lenguaje de controladores lógicos programables (PLC) y un sistema de supervisión y adquisición de datos (SCADA) para esa instalación docente. Utiliza herramientas profesionales para modelar, verificar y validar la propuesta de automatización, donde se adicionan dos nuevos pasos para buena formación de la PN, y para la programación, comunicación y supervisión de la aplicación. Con la puesta en marcha de la instalación de laboratorio se cuenta con una herramienta cercana a un sistema de automatización real, utilizable en varias asignaturas de pre y postgrado. El uso del modelado formal, la comunicación OPC y el diseño del supervisorio permiten la puesta a punto de la aplicación y constituye un ejemplo a seguir en la enseñanza de la Automática.

Palabras-clave: Modelado en Redes de Petri, Comunicación OPC, Supervisión industrial, Enseñanza de Automatización.

\section{INTRODUCCION}

Desde el siglo pasado, en disímiles industrias destinadas a la fabricación y embalaje de materiales sólidos y productos alimenticios se propuso insertar un proceso de llenado de vagones que permitiera solucionar el retraso en el transporte y dosificación de diferentes materiales granulados. Este proceso consta de diferentes topologías en dependencia del área en que se encuentre [13].

Este proceso de llenado de vagones es de gran importancia en las industrias de construcción, azucarera, ITEGAM - JETIA Vol. 01, No. 01. Março de 2015. Manaus - Amazonas, Brasil. ISSN 2447-0228 (ONLINE). niquelífera, minera, etc. Su objetivo es mantener un correcto suministro de materias primas en un vagón, para posteriormente ser transportado o utilizado en diferentes procesos, en dependencia de la industria.

El proceso de llenado de vagones consta de diferentes etapas como son: almacenamiento del material, suministro del material a la cinta transportadora, transporte del material a través de la cinta y pesaje del material en el vagón. Con el correcto diseño de control de estas etapas, teniendo en cuenta las diferentes especificaciones que requiere el proceso, se puede lograr un correcto funcionamiento. Debido a la importancia de este proceso, el departamento de Control Automático de la 
Israel F. Benítez-Pina, Michel Sanz-Pérez, Jose Rogelio Martin-Escalona/ITEGAM-JETIA.Vol.01, No 04, pp.25-34. Dezembro, 2015.

Facultad de Ingeniería Eléctrica en la Universidad de Oriente determinó diseñar una maqueta educativa del proceso. El objetivo de este trabajo se centra en realizar el diseño de un sistema de enseñanza que incluye maqueta, sistema de supervisión y adquisición de datos (SCADA) [1, 2, 4, 19] y programación de controlador lógico programable (PLC) [16] para el sistema de automatización integrada de un proceso de llenado de vagones de dosificación de material de construcción.

Se propone el estudio y la obtención de un modelo en Redes de Petri [17] del proceso de llenado de vagones, para su posterior traducción en lenguaje de programación y creación de un SCADA para dicha práctica de laboratorio.

Para el desarrollo del trabajo fueron empleados el Visual Object Net 2.7 [8], MicroWinV4.0, servidor OPC KEPware, y el EROS 5.10 para modelar, verificar y validar la propuesta de automatización, y luego la programación, comunicación y supervisión de la aplicación respectivamente.

En el punto 2 se tratan los elementos teóricos principales que permiten el desarrollo del trabajo. En el punto 3 se explica el modelado del programa principal destacando su verificación y validación formal. En el punto 4 se trata el modelado y comportamiento del sistema en funcionamiento automático desde el control local hasta la supervisión. Como conclusión se valoran los resultados obtenidos.

\section{BASES TEÓRICAS DE LA INSTALACIÓN DE AUTOMATIZACIÓN DEL PROCESO DE LLENADO DE VAGONES}

En este epígrafe se hace una exposición de los diferentes aspectos teóricos tenidos en cuenta a la hora de realizar el presente trabajo, como: descripción del flujo productivo de la planta, modelación de Redes de Petri, programación simulada y la real sobre el PLC incluyendo un servidor para la gestión de la comunicación, sistema de supervisión e implementación del sistema de automatización integrada.

\section{II.1 DESCRIPCIÓN DEL FLUJO PRODUCTIVO DEL SISTEMA}

De acuerdo a la importancia del proceso de llenado de vagones, se hace un estudio de la operación del proceso.

La planta de llenado de vagones se puede dividir en cuatro etapas:

- Almacenamiento: cuenta con una tolva principal en la cual se almacena la materia prima (sólido granulado) que va a ser suministrada al vagón.

- Suministro: se lleva a cabo a través de una compuerta. El movimiento de la compuerta de salida del material de la tolva se realiza por medio de un cilindro neumático de simple efecto que se acciona por una electroválvula $3 / 2$ vías.

- Transporte: el material llega al vagón mediante una cinta transportadora que es accionada por un motor trifásico.
- Pesaje: el vagón se monta sobre una plataforma, que está soportada sobre sensores de carga que son los encargados de medir el peso del material que está siendo suministrado a este

La Figura 1 ilustra una maqueta del proceso con el panel de comando manual y el PLC utilizado en el control local.

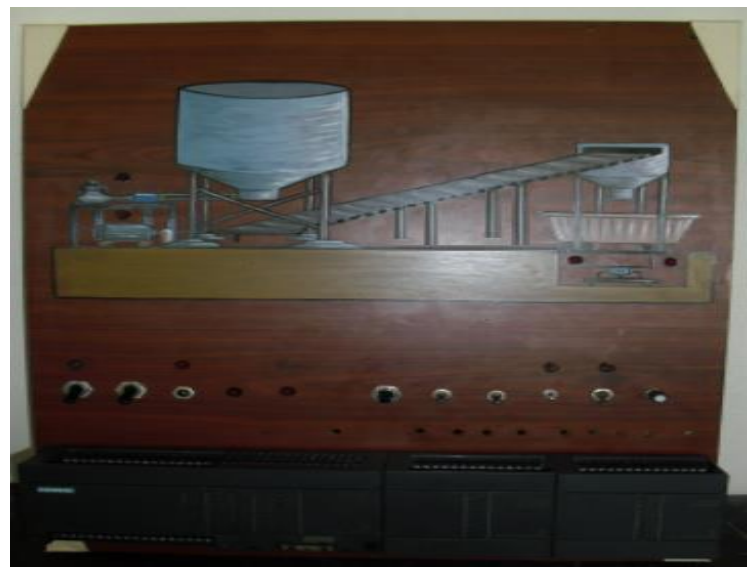

Figura 1. Maqueta del llenado de vagones y PLC de control local [13]

Luego de analizadas las diferentes etapas de este proceso, para el diseño del sistema de automatización del mismo, se hace un estudio del modo de operación del proceso utilizando el modelado formal [10] de acuerdo a los resultados del estudio realizado del estado del arte en diseño de la automatización industrial.

\section{II.2 MÉTODOS DE DISEÑO FORMAL DE SISTEMAS AUTOMÁTICOS}

La automatización de sistemas industriales modernos, tiene un fuerte componente definido por la coordinación de las actividades de los diferentes subsistemas, que se integran en las redes de control distribuido industrial que se utilizan actualmente. La coordinación de esas actividades se torna más compleja en la medida que se incrementan las posibilidades de desarrollar diferentes producciones, en el mismo sistema productivo. Esto garantiza el nivel de atención al cliente, que requiere de un comercio electrónico competitivo moderno. Por tanto, la proyección de sistemas de control industrial incrementa su complejidad y necesita de métodos formales para el modelado de estos sistemas [3]

Para el diseño de estos sistemas de automatización industrial integrada que tienen alta complejidad por la sincronización de múltiples funciones, las redes de Petri constituyen uno de los formalismos más aceptados por sus potentes teorías de análisis y síntesis y una directa representatividad grafica. [7]. 
Israel F. Benítez-Pina, Michel Sanz-Pérez, Jose Rogelio Martin-Escalona/ITEGAM-JETIA.Vol.01, No 04, pp.25-34. Dezembro, 2015.

Las etapas de desarrollo de cualquier automatización moderna incluyen [4]:

1. Diseño: donde se requiere hacer el análisis y síntesis del sistema automatizado. Esto es solo posible con el uso del Modelado Formal de la planta industrial y de sus especificaciones de seguridad y comportamiento como sistema controlado.

2. Programación: los modelos deben ser programados en lenguajes normalizados de automatización con facilidades de simulación de su funcionamiento. Como los PLC's son los dispositivos de mayor uso, la IEC61131 es la norma más usada, así como sus ambientes de edición y simulación.

3. Implementación: para ello se requiere crear estructuras jerárquicas en redes buscando eficiencia y facilidades de ampliación, modificación y mantenimiento para reducir tiempo y costos en el proyecto, y luego en el funcionamiento de la empresa.

En la primera etapa se requiere hacer el análisis y síntesis del sistema automatizado. Para ello se modela la planta, teniendo en cuenta sus especificaciones de seguridad y comportamiento como sistema controlado. Al cual se le realiza la verificación de sus propiedades principales y validación del cumplimiento de las exigencias del cliente, por medio de la simulación del funcionamiento de la planta con su control integrado. Por tales motivos el mundo académico e industrial [3, $6,7,9,10,11,12,14,16,20]$ reconoce que solo modelando se logra la implementación de sistemas de automatización eficientes.

Es preciso mencionar que existe un proyecto europeo que tiene como perspectiva una integración de las metodologías de modelado, diseño e implementación de sistemas de automatización, el MEDEIA FP7-2207-211448 [9]. Este describe el concepto de Dominio de Vistas Específicos (DSVs), de acuerdo con las especificaciones del comportamiento controlado que provee el desarrollo del control. El proyecto MEDEIA intenta definir un mega modelo (ver figura 2) para guiar el diseño de cualquier sistema de automatización. En la bibliografía [9] se presentan tres áreas de automatización: Manufactura, Robótica e Energía. Estos DSVs generan informaciones para desarrollar un Componente de Automatización en el ACIM (Modelo de implantación de componentes de automatización).

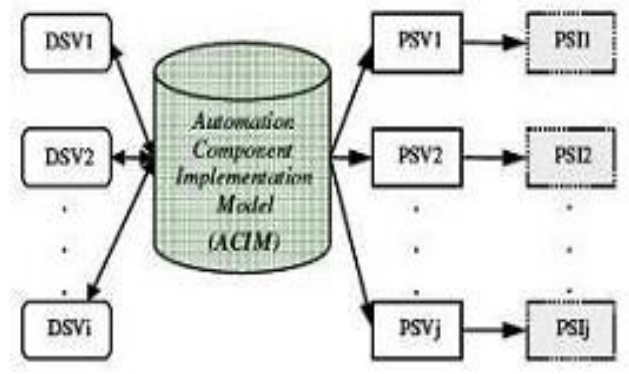

Figura 2. Mega modelo para la automatización [9].

La proyección de sistemas de automatización parte de las especificaciones de control y comportamiento; además teniendo en cuenta algunas de las especificaciones mecánicas, eléctricas y funcionales (como las señales de entrada y salida de los PLC's, los esquemas eléctricos y limitaciones estructurales), para completar la información requerida para el modelado del sistema. Luego se trabaja en un modelo de planta, para crear un modelo de diagnóstico y comportamiento del sistema controlado.

Precisamente los DVS relacionados con especificaciones mecánicas, eléctricas y funcionales fueron considerados en todo el diseño de este trabajo.

El diseño de la automatización industrial moderna está encausado a la creación de sistemas complejos con gran intercambio entre sus partes y alto grado de soluciones programables; por tanto, se requiere realizar la verificación formal [14] del desarrollo de ese software para garantizar cumplir con los objetivos del sistema controlado, con eficiencia y seguridad del funcionamiento. La importancia del proceso de Verificación y Validación $(\mathrm{V} \& \mathrm{~V})$ fue reconocida en la ingeniería de software desde sus inicios [14]. La programación del sistema es en esencia una tarea de solución de problemas e implica la validación de la solución establecida en combinación con la verificación del modelo.

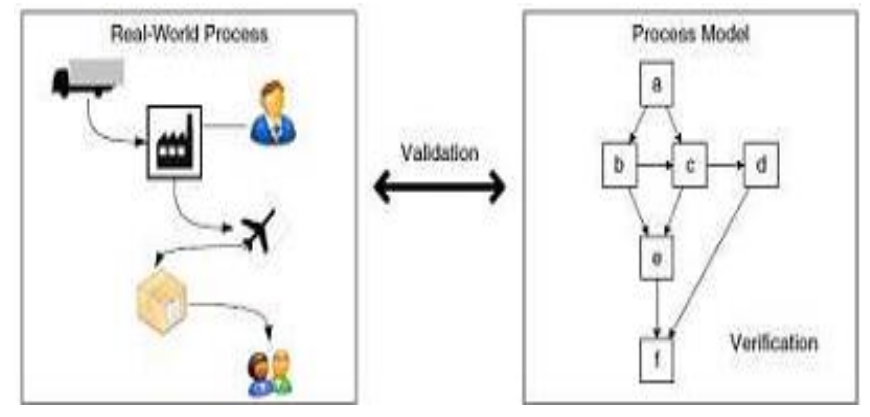

Figura 3. Esquema de V\&V en un Modelo y Realidad de un proceso [14].

Verificación: está orientada a las propiedades generales del modelo y a la satisfacción de una fórmula dada por el modelo (ver parte derecha de la figura 3 ).

Validación: aborda la consistencia del modelo con el universo del discurso, o sea, el proceso del mundo real (ver centro de la figura 3 ).

En [14] se realiza un análisis comparativo de varios métodos formales y brinda siete pasos que deben ser realizados en cualquier proceso de modelado para garantizar la reducción de los errores, los cuales se muestran a continuación:

- Usar el mínimo de elementos posible en el modelo.

- Minimizar las rutas por elementos.

- Utilizar una entrada y una salida.

- Modelar lo mas estructurado posible.

- Evitar rutas OR (selección aleatoria).

- Colocar el máximo de comentarios funcionales.

- Descomponer modelos con más de 50 elementos.

Basado en la experiencia practica que tenemos, a estos pasos adicionamos otros dos pasos de relevante importancia para 
Israel F. Benítez-Pina, Michel Sanz-Pérez, Jose Rogelio Martin-Escalona/ITEGAM-JETIA.Vol.01, No 04, pp.25-34. Dezembro, 2015.

garantizar buena formación de los modelos:

- Cuando se bifurca la secuencia de operación por caminos alternativos, estos caminos deben unirse de la misma forma.

- Cuando se bifurcan caminos paralelos deben cerrarse con una sincronización de todos esos caminos paralelos.

Con esto se completa la coherencia del modelo garantizando buena formación que permita mejorar la vivacidad del modelo.

En este trabajo el proceso de modelado se realizó cumpliendo los siete pasos [14] ampliados para el modelado de procesos, pues es desarrollado de forma modular. Las secuencias fueron minimizadas para atender los objetivos de cada secuencia de la forma más rápida posible, todas las subredes tienen una entrada y una salida. Los modelos fueron creados manteniendo buena formación de todas sus redes, principalmente evitando mezclar secuencias paralelas con secuencias condicionales. Esto garantiza un alto por ciento del cumplimiento de las propiedades verificables en el modelo.

El proceso de $\mathrm{V} \& \mathrm{~V}$ en este trabajo se realiza con métodos de verificación de buena formación de las redes y por vía de la simulación que garantizan el cumplimiento de las propiedades fundamentales del modelo y de los requisitos funcionales. Esto junto a su traducción a un programa del PLC asegura un funcionamiento eficiente del sistema automatizado.

Los métodos de simulación [16] fueron desarrollados para estudiar una gran variedad de aspectos sobre el sistema real, como son: su comportamiento bajo los parámetros de operación del sistema, impacto de los cambios en su desempeño, así como estudiar sistemas que están todavía en la fase de concepción y como una herramienta de proyecto para evaluar y validar el desempeño de nuevos sistemas.

La mayor disponibilidad de herramientas de simulación, la creciente capacidad computacional, y los avances en las metodologías de simulación convirtieron a la simulación en una de las técnicas más usadas y aceptadas en tareas de análisis y desarrollo de sistemas [16].

Del estado del arte internacional además se conoce que los sistemas de automatización industrial pueden dividirse en dos niveles básicos de automatización [5], y de igual forma los métodos de modelado específicos. Estos son:

1. Sistemas Supervisorios: generalmente desarrollados en niveles superiores de redes de automatización industrial, utilizando sistemas de desarrollo de SCADA profesionales. En el modelado de sistemas supervisorios son utilizados métodos y herramientas de desarrollo de software. El UML 2.2 [18] es un estándar internacional en el área, especialmente en la primera etapa de diseño.

2. Automatización Local: son utilizadas tecnologías programables en redes de PLCs con capacidad de controlar redes de campo de sensores y actuadores inteligentes, una interfase hombre-máquina local y comunicaciones Ethernet Industrial para así enlazar con un Supervisorio del sistema de automatización. La programación de los PLCs es estandarizada por la norma IEC61131 y la metodología de diseño más práctica para relacionarse con esto es la basada en Sistemas de Eventos Discretos (SED) con Redes de Petri (PN) [20].
Las principales ventajas de utilizar PN [17] como herramientas de modelado de un SED las brinda su fundamentación matemática, representación compacta del comportamiento del sistema para lo que es preciso modelar los estados que son importantes para la correcta secuencia lógica del sistema; naturaleza gráfica con simulación que ayuda en la comprensión de sistemas complejos, pues la simulación resulta simple y práctica para encontrar y corregir inconsistencias en el modelado, resultando las $\mathrm{PN}$ una opción útil para describir y estudiar sistemas que se caracterizan por ser concurrentes, asincrónicos, distribuidos, paralelos, no determinísticos y/o estocásticos.

En el análisis de modelos PN de sistemas de automatización, es muy importante la propiedad de Alcanzabilidad de estados, pues a partir de un estado inicial muchas veces se requiere que la red avance automáticamente hasta un estado dado, si no tiene esta capacidad, no podrá ejecutar el comportamiento deseado. A esto está relacionada la Vivacidad de la red, si generalizamos esta capacidad a todo el sistema, y es aquí donde pueden detectarse partes de la red que detienen su funcionamiento (como autolazos, bloqueos,) lo cual nos permite eliminar estas situaciones anormales en el programa del PLC desde esta etapa inicial de diseño.

Existen innumerables publicaciones sobre el uso de las PN como herramienta de modelado de un SED y trabajos desarrollados principalmente en industrias donde se hace uso de las PN [20].

En [20], se valora la mejor opción entre estos modelos y se define la PN GHENeSys como una 6-tupla:

$$
\mathrm{N}=(\mathrm{L}, \mathrm{A}, \mathrm{F}, \mathrm{K}, \mathrm{M}, \Pi, \mathrm{Q})
$$

Donde:

- Los elementos $\mathbf{L}$ son llamados Lugares $(\mathbf{L}=\mathbf{B} \cup \mathbf{P})$; el subconjunto B (Boxes) son lugares normales enlazados por arcos normales, y los $\mathbf{P}$ (Pseudoboxes) son lugares de marcación permanente $\{0,1\}$ que solo son modificados por eventos externos, siendo enlazados con transiciones controladas por medio de arcos habilitadores e inhibidores.

- A es el conjunto de las transiciones (llamadas Actividades).

- $\mathbf{F}$ son las relaciones de flujo $(\mathbf{F} \subseteq(\mathbf{L} \times \mathbf{A}) \cup(\mathbf{A} \times \mathbf{L}))$.

- $\mathbf{K}: \mathbf{B} \rightarrow \mathbf{N}+$, es la función de la capacidad de los Boxes.

- $\mathbf{M}: \mathbf{L} \rightarrow \mathbf{N}+$ es la marcación inicial de la red.

- I es una función que diferencia elementos simples ( $\mathbf{L}$ y A, atribuyendo el valor 0), de los macro elementos (elementos que representan subredes; atribuyendo el valor 1).

- $\mathbf{Q}$ es una función que asocia arcos de nivel (atribución) para algunos grupos (B) o arcos de impulso (set o reset) para el disparo de algunas Actividades (A).

En las redes GHENeSys se hacen mejorías a las PN ordinarias, garantizando el enlace con las salidas de los PLC por el uso de la función $\mathbf{Q}$, y posibilitando la creación de macro elementos (subsistemas que pueden ser reutilizados) y la utilización de lugares auxiliares (Pseudoboxes ligados a sensores e informaciones externas la red) como habilitadores e inhibidores de transiciones por medio de los arcos respectivos [20]. 
Israel F. Benítez-Pina, Michel Sanz-Pérez, Jose Rogelio Martin-Escalona/ITEGAM-JETIA.Vol.01, No 04, pp.25-34. Dezembro, 2015.

A continuación se aplica esta teoría al sistema de llenado de vagones para dosificación de áridos.

\section{AUTOMATIZACIÓN LOCAL PARA EL PROCESO DE LLENADO DE VAGONES}

La maqueta que se construyo para estas prácticas de laboratorio tiene las siguientes dimensiones $95 \times 45 \times 5 \mathrm{~cm}$ (ver Fig. 1). Está conformada por un sinóptico del proceso que es controlado por un PLC S7-200 de SIEMENS, siete interruptores con los cuales se actúa para simular entradas discretas que exige el proceso como son; encendido (ON_OFF), manual-automático, tolva en posición, compuerta, cinta transportadora, comprobar cinta, comprobar compuerta, un Push-Booton para simular emergencia, y un potenciómetro para simular el peso del vagón (entrada analógica al PLC). La indicación de las salidas, se visualiza a través de ocho diodos Led.

El desarrollo de la secuencia se arranca y se para con un pulsador (enciende o apaga una lámpara). Una vez que la secuencia ha sido iniciada, se conecta la cinta transportadora con un contactor si el vagón se encuentra en la posición de llenado (final de carrera). La cinta transportadora se para nuevamente cuando el vagón ha abandonado la posición de llenado y no ha llegado otro vagón vacío en el transcurso de 20 segundos. La compuerta se abre cuando la cinta marcha y existe un vagón vacío (peso aun no alcanzado) en la posición de llenado. Esta se cierra nuevamente cuando la báscula alcanza el peso ajustado.

Las órdenes de apertura y cierre de la compuerta no tienen por qué permanecer aplicados durante el tiempo que esta tarda en desplazarse hacia su nueva posición. Para conseguir que el material existente en la cinta se descargue sobre el vagón después del cierre de la compuerta, el depósito de bloqueo se abre 10 segundos después que en la báscula se ha alcanzado el peso. El dispositivo de bloqueo se cierra nuevamente cuando el vagón lleno ha abandonado la posición de llenado, con lo cual se abre nuevamente el contacto final de carrera. Cuando el vagón siguiente alcanza la posición de llenado, se repite el proceso descrito hasta que se bloquea el desarrollo del mando con el pulsador de apagado.

El proceso del llenado de vagones se modeló completamente en PN. Se realizaron cuatro subredes utilizando el programa Visual Objet Net 2.7 (VON, [8]) las cuales corresponden a un programa principal, funcionamiento manual, funcionamiento automático, detección de alarmas y detección de fallos. En las subredes realizadas se da cumplimiento a los requerimientos funcionales del sistema. El uso del programa VON permite la simulación de todos los modelos realizados, proceso de suma importancia para la validación.

\section{III.1 MODELADO EN REDES PETRI DEL PROGRAMA PRINCIPAL}

En este programa principal se define el arranque y modo de operación del sistema de control automatizado. En la figura 4 se muestra el modelado del Programa Principal hecho en PN utilizando el programa VON.

En este modelado interactúa el subsistema Comportamiento Funcional del Sistema (CFS), que es afectado por los estados de diferentes variables del sistema como son las representadas por los lugares normales y auxiliares: "Inicio", "Parado" "ON/OFF", "Emergencia", "Manual/Automático". El subsistema CFS cuenta con cincos estados en los que el funcionamiento del sistema se puede encontrar, los cuales están representados como dos lugares simples, "Parado" e "Inicio", junto a tres subredes o macro-lugares, "MANUAL" (P6), "AUTOMÁTICO" (P5) y "EMERGENCIA" (P7).

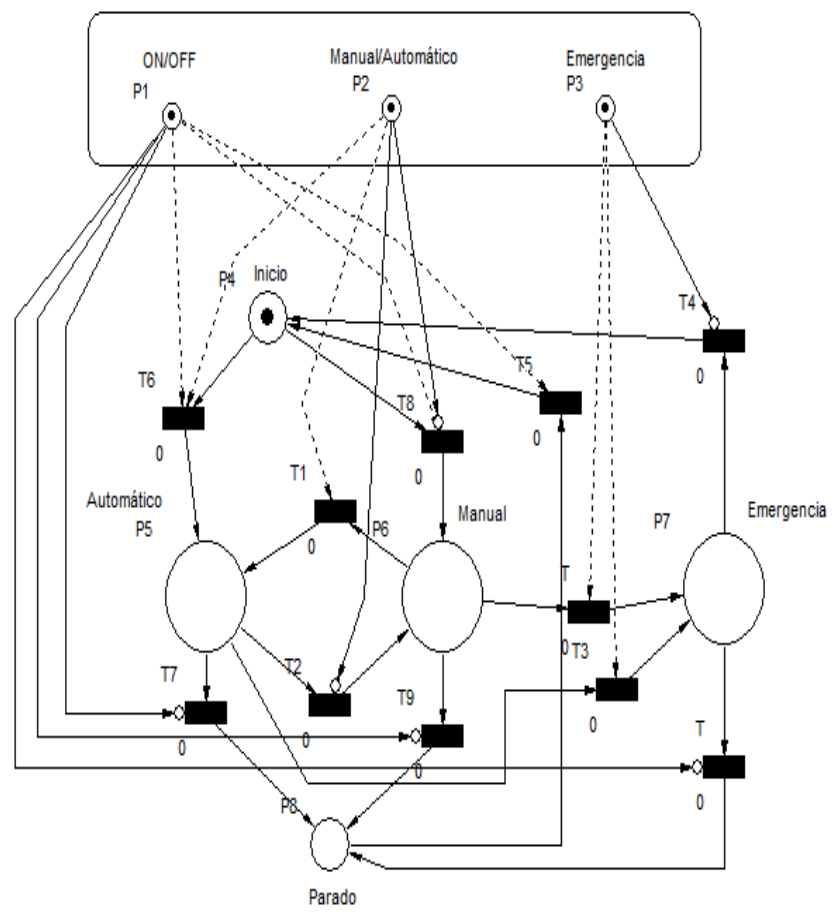

Figura 4. Modelado en Redes Petri del programa principal

El proceso comienza una vez que es conectado el voltaje de alimentación, actuando sobre el interruptor correspondiente a 'ON/OFF'. Una vez encendido, comienza la ejecución de las diferentes etapas del proceso. $\mathrm{Si}$ el selector de modo de operación no está activo el proceso se inicia en modo "Manual" por defecto. Si se desea cambiar a modo "Automático" se debe actuar sobre el selector de modo una vez. Si en la ejecución del proceso se produjera una situación que demande la detección urgente del proceso, el panel cuenta con un botón de "Emergencia" el cual detiene el proceso en su totalidad.

La red de Petri autónoma subyacente en el modelo interpretado de la Figura 4, se representa en la Fig. 5 en la que fueron comprobadas las propiedades de Alcanzabilidad y Limitación estructural, quedando disparabilidad y controlabilidad auto-contenidas en las otras dos propiedades. El método de reducción [17] es el más simple para el cumplimiento de estas propiedades. Se demuestra fácilmente que toda la red puede ser reducida a un lugar y una transición cumpliendo las propiedades contenidas en redes bien formadas [17].

La simulación dinámica de la red interpretada valida los requisitos funcionales y demuestra la ausencia de bloqueos y su capacidad de retornar al estado inicial. 
Israel F. Benítez-Pina, Michel Sanz-Pérez, Jose Rogelio Martin-Escalona/ITEGAM-JETIA.Vol.01, No 04, pp.25-34. Dezembro, 2015.

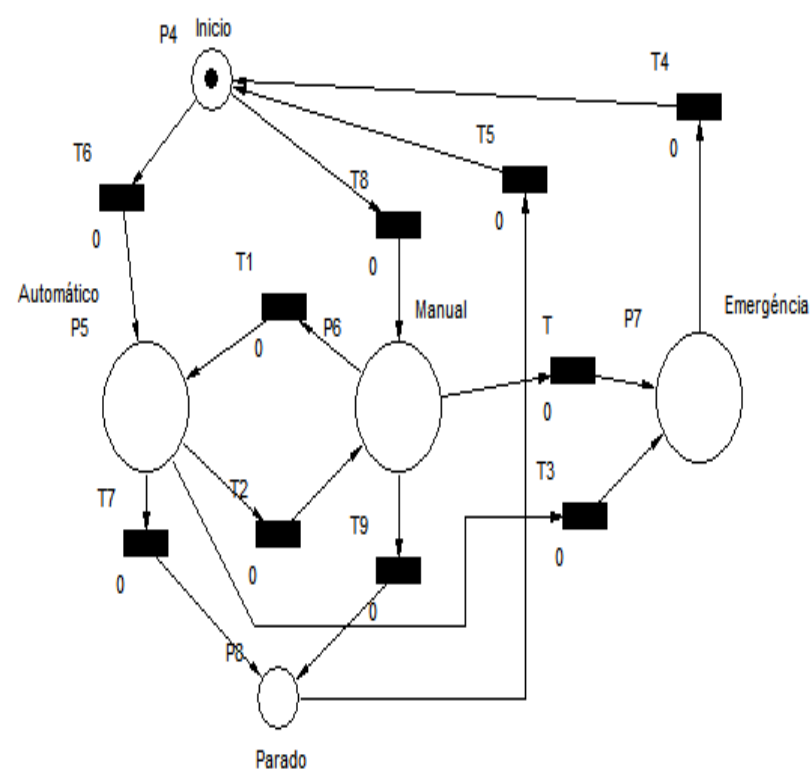

Figura 5. Red de Petri Autónoma del Modelado del macro lugar "Func Auto" del Sistema de Control Automatizado propuesto.

En más detalles se evalúa el comportamiento de la subred de funcionamiento automático (Marcolugar P5), pues el accionamiento manual y el de emergencia son redes mucho mas sencillas que el automático.

\subsection{Modelado en Redes Petri del funcionamiento en automático del macro lugar "P5".}

El macrolugar "AUTOMATICO", es donde se desarrolla todo el funcionamiento automático del sistema de control automatizado propuesto, como está representado en la figura 6. Este funcionamiento automático opera de forma cíclica. De esta forma en esta red se puede apreciar la existencia de conflictos importantes de sincronización de los subsistemas integrantes, los que son resueltos adicionando arcos y lugares auxiliares.

El proceso parte de la existencia de la marcación en el panel de control en "Automático", una vez en el modo manual se hace el "Chequeo de Fallo y de Alarmas" para determinar posibles irregularidades en el funcionamiento del proceso. Luego de determinada la no existencia de fallos y alarmas se pasa a la ejecución de las secuencias del proceso:

- ¿Hay vagón en posición de llenado?.

- Si hay vagón en posición, encender cinta transportadora.

- Abrir la compuerta de suministro de materiales.

- Censar el peso del vagón en la plataforma de pesaje.

- Cuando se alcance el valor de pesaje deseado cerrar la compuerta.

- Habilitar el bloqueo del vagón, durante 10 segundos y se deja la cinta transportadora encendida.

- Liberar el vagón lleno de la plataforma de pesaje.
- Si no se posiciona en la plataforma de pesaje un nuevo vagón en un intervalo de tiempo de 20 segundos se detiene la cinta transportadora.

Luego se aplica la misma metodología del caso anterior y se verifica el cumplimiento de varias propiedades de las PN como son la limitabilidad, alcanzabilidad, disparabilidad limitada estructural y controlabilidad. Para esto se obtiene la red de Petri autónoma subyacente en el modelo eliminando lugares y arcos auxiliares, lo que demuestra que esta constituida por 4 subredes independientes.

Todas son redes secuenciales muy simples que cumple con la condición de tener todos los lugares iniciales marcados [17], por lo que el cumplimiento de las propiedades estructurales y comportamentales es evidente.

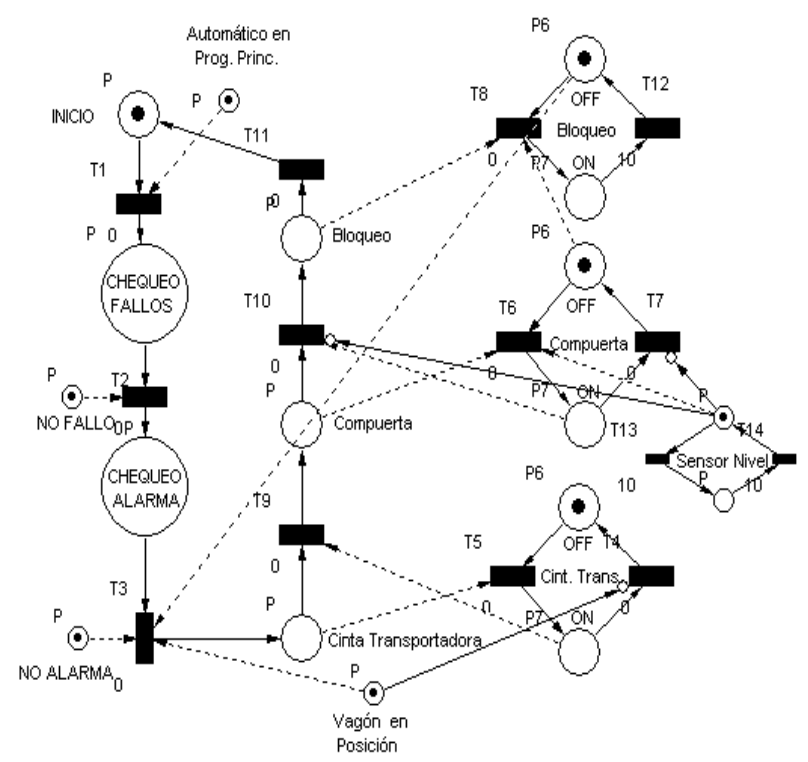

Figura 6. Modelado en Redes Petri del funcionamiento automático del llenado de vagones

Además la simulación dinámica de la red bajo diferentes condiciones permite validar el cumplimiento de los requisitos funcionales de este sistema.

Luego se comprobado el modelado del sistema se pasa a la fase de programación definiendo el hardware de implementación para mayor compatibilidad con la última fase.

$\mathrm{El}$ autómata programable (PLC) empleado en el diseño del control pertenece a la familia de S7-200, específicamente CPU 214. El MicroWinV4.0 es el software que permite la programación de esta familia de PLC's. Dentro de sus principales ventajas se encuentra el simulador de PLC en PC, que permite depurar los programas para el control de procesos antes de su implementación. Mediante este software se realizó toda la programación del PLC teniendo en cuenta las entradas y salidas del proceso y las especificaciones funcionales y de control del mismo verificadas y validadas mediante el modelado formal explicado en el epígrafe anterior. El lenguaje de programación empleado fue el LD (Ladder Diagram), debido a que genera un programa más compacto de fácil comprensión, y además es muy 
Israel F. Benítez-Pina, Michel Sanz-Pérez, Jose Rogelio Martin-Escalona/ITEGAM-JETIA.Vol.01, No 04, pp.25-34. Dezembro, 2015.

sencilla su modificación en caso de ampliación, cambio o mantenimiento. También este lenguaje tiene un conjunto de analogías con los modelos en GHENeSys (explicados en [20]) que permiten una fácil traducción de los modelos a programas IEC61131 compatibles.

Se tuvo en cuenta las protecciones necesarias en caso de una emergencia, así como los modos de funcionamiento del proceso. El funcionamiento manual/automático del proceso, se decide a través de una variable booleana declarada en el PLC correspondiente a un interruptor de entrada de dos posiciones. En modo manual el operador manipulará el proceso tomando sus propias decisiones, y en el modo automático el PLC controla el proceso siguiendo el control programado. El programa resultante fue validado mediante simulación reduciendo las posibilidades de errores de diseño que ya habían sido minimizados previamente mediante el modelo formal.

\section{SISTEMA SUPERVISORIO PARA EL PROCESO DE LLENADO DE VAGONES}

Un sistema de supervisión y control permite que un operador ubicado en una estación central, incluso a grandes distancias de la ubicación de los procesos industriales, pueda hacer ajustes o cambios en los controladores locales de los procesos. En este caso es importante conocer el estado de los interruptores de seguridad de un sistema, monitorear el estado de las alarmas del proceso y obtener información de las variables del proceso involucradas, tanto para realizar análisis $\mathrm{y}$ comparaciones estadísticas para informes productivos y la toma de decisiones. El software utilizado en este proceso fue el SCADA cubano Eros 5.10.

\section{IV.1 CONTROL DE ACCESO EN EL SUPERVISORIO}

En el SCADA se hace una verificación del control de acceso (ver figura 7), esto se debe a que no todos los usuarios pueden tener el mismo nivel de acceso. En el supervisorio EROS se establece la jerarquía, que es el derecho que tendrá el usuario para acceder a las diferentes opciones del sistema, este valor va de 0 a 255 donde 0 define derechos de invitado y 255 todos los derechos y es por ello el que el administrador posee por omisión.

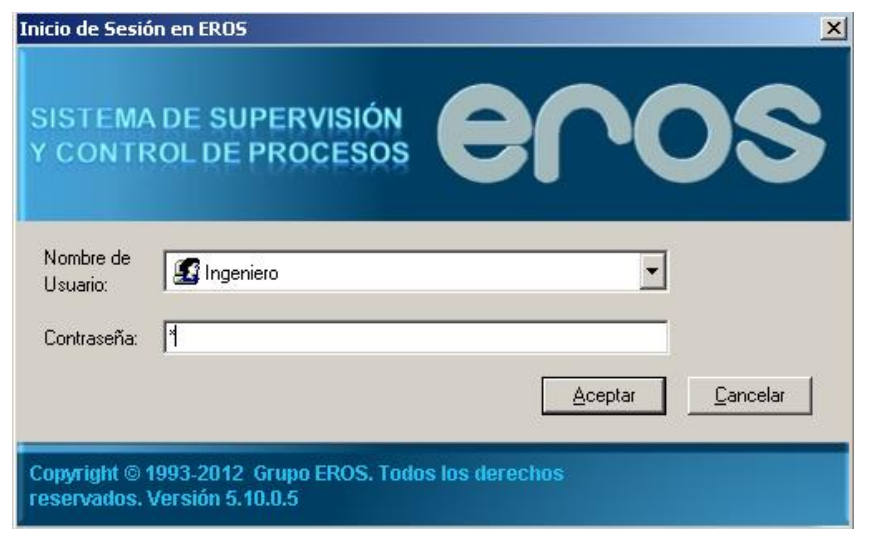

Figura 7. Nivel de acceso al sistema de supervisión.
La seguridad de un SCADA industrial cuenta con cinco niveles de acceso para determinados usuarios, en caso de que se desee manipular alguna variable. Estos niveles son:

- Operador: Solo puede modificar los parámetros a los cuales esté autorizado por el Ingeniero, por defecto el SCADA arranca en esta sesión. La jerarquía de este es de 125.

- Ingeniero: Tiene todos los privilegios sobre el SCADA. En esta sección se puede manipular todas las variables del proceso, hacer ajuste de parámetros y variar los diferentes mímicos. La jerarquía de este es de 255 .

- Gerente: Este nivel solo tiene acceso a visualización, históricos y reportes, debido a que el gerente es quien debe llevar las estadísticas y de hacer los diferentes balances que se llevan en la industria con el fin de determinar la eficiencia de la productividad. La jerarquía de este es de 10.

- Invitado: Este solo tiene acceso a visualización, por defecto el SCADA arranca en esta sección. La jerarquía de este es de 0 .

- Administrador: En esta sección se tiene todo los privilegios por lo cual a través de esta se puede configurar las aplicaciones pertinentes. La jerarquía de este es de 255

En esta instalación docente solo se requiere de los niveles 1, 2 y 5 del EROS asociándolas a estudiante, profesor y técnico del laboratorio respectivamente.

\section{IV.2 CHEQUEO DE ALARMAS EN EL SUPERVISORIO.}

Las alarmas o avisos son de gran importancia ya que garantizan la comunicación necesaria entre el operario y el sistema, y además ayudan a la toma de decisiones. A través de ellas se informa acerca de los estados de funcionamiento y de fallo que se producen durante el proceso. Sirven para poder detectar con antelación situaciones críticas y para evitar períodos de inactividad. Al configurar las alarmas se definen qué eventos activarán las mismas, y si estarán activas hasta que alguien las solucione.

En el EROS se pueden configurar de varias formas en dependencia del tipo de variable que se desee. Los tipos de alarmas son:

- Chequea Razón de Cambio: Establece si se va a chequear la alarma por razón de cambio. Para ello hay que definir el cambio que no debe sufrir el valor de la variable (por encima o por debajo), de una medición a otra, o sea una variación brusca de tendencia.

- Chequea Alarma Prohibitiva: Define si se va a chequear alarma prohibitiva o no y se detallan los rangos fuera de los cuales la variable puede provocar una avería o un deterioro del material que se procesa o es totalmente 
Israel F. Benítez-Pina, Michel Sanz-Pérez, Jose Rogelio Martin-Escalona/ITEGAM-JETIA.Vol.01, No 04, pp.25-34. Dezembro, 2015.

anormal que trabaje en esos valores y en los cuales debe trabajar la alarma sonora.

- Chequea Alarma de Operación: Define si se va a chequear alarma de operación o no y se detallan los rangos fuera de los cuales la variable se está desviando de los parámetros de operación normales.

- Chequea Alarma en OFF: Define que la alarma se indicará cuando la señal está en off.

- Chequea Alarma en ON: Determina que la alarma se indicará cuando la señal está en on, es decir, invertida.

En el diseño de la maqueta se tuvo en cuenta las alarmas de tipo "ON" y "Prohibitivas", en la tabla 1 se encuentra la relación de las alarmas que fueron declaradas en el proceso de la maqueta de laboratorio.

Tabla 1 Alarmas del proceso.

\begin{tabular}{|l|c|c|}
\hline \multicolumn{1}{|c|}{ Nombre } & $\begin{array}{c}\text { Alarma } \\
\text { ON }\end{array}$ & $\begin{array}{c}\text { Alarma } \\
\text { Prohibitiva }\end{array}$ \\
\hline Alarma_Peso_Alto & $\mathrm{X}$ & $\mathrm{X}$ \\
\hline Alarma_Compuerta & $\mathrm{X}$ & $\mathrm{X}$ \\
\hline Alarma_Cinta & $\mathrm{X}$ & $\mathrm{X}$ \\
\hline Fallo_Cinta & $\mathrm{X}$ & $\mathrm{X}$ \\
\hline Fallo_Compuerta & $\mathrm{X}$ & $\mathrm{X}$ \\
\hline
\end{tabular}

Una vez que son configuradas, se pasa al modo de operación, y cuando se ejecutan se pueden observar en una tabla que establece el supervisorio (ver figura 8) para registrar las alarmas, donde se almacena la fecha y hora que se produjo y si se dió atención a la misma.

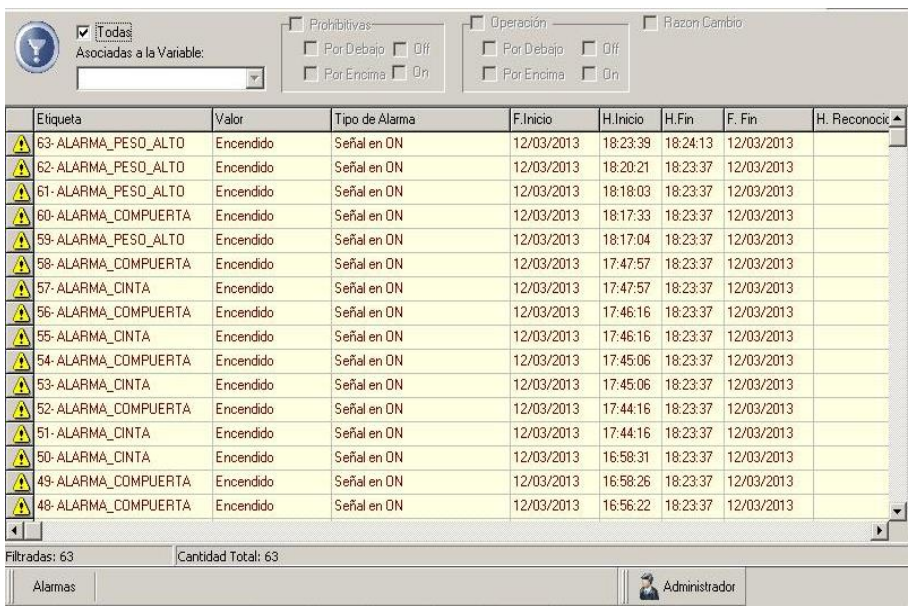

Figura 8 Registro de alarmas.

\section{IV.3 DISEÑO DE MÍMICOS O SINÓPTICOS}

Los mímicos o sinópticos son dibujos que representan un área del proceso y en ellos se muestra el estado de los equipos, los valores de las variables, la posición de algún aditamento, imitaciones de instrumentos o animaciones de equipos con el objetivo de mostrar la información que se mide de una manera más rápida, sencilla y agradable a la vista del operador evitando afectaciones por fatiga o lentitud de manipulación del operador.

La ventana principal para la supervisión del proceso se muestra en la figura 9. En la pantalla se pude observar el comportamiento real del proceso. Se visualizan además, las señales provenientes de los sensores y otras variables del programa.

Se tiene también la actualización de la hora y la fecha en tiempo real, esto permite hacer registros actuales, con el fin de detectar fallas en el proceso para agilizar la toma de decisiones. Estas fallas pueden ser por el mal funcionamiento del mismo o por la mala manipulación de un operador en su turno laboral.

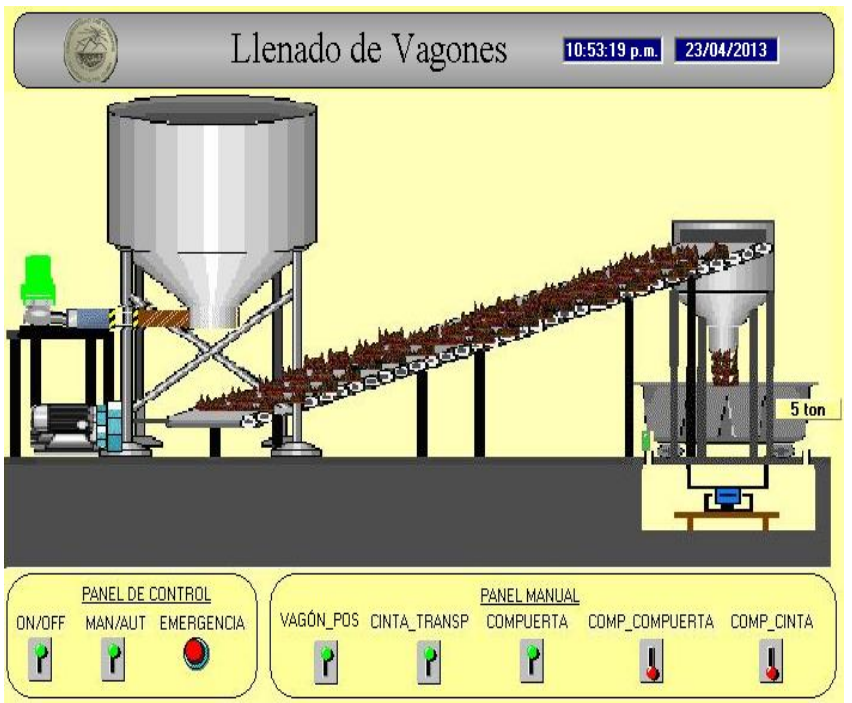

Figura 9 Pantalla principal del processo.

La pantalla para la supervisión del proceso tiene un panel de control, mostrado en la figura 9 (lateral inferior izquierdo), donde se muestran los controles que intervienen en el proceso. Se podrán modificar desde la pantalla la forma en que opera el sistema, si es en manual o automático, se podrá energizar el proceso, además, se incluye un botón de Emergencia en caso que se produzca alguna situación indeseable.

La pantalla para la supervisión del proceso también cuenta con un panel manual, mostrado en la figura 9 (lateral inferior derecho), donde se muestran una serie de controles que intervienen en el proceso con el fin de operar de forma manual el proceso.

\section{IV.4 SERVIDOR KEPWARE-OPC PARA LA GESTIÓN DE LA COMUNICACIÓN}

Un servidor OPC es un estándar abierto de comunicación en el campo del control y supervisión de procesos industriales que ofrece un interface común para la comunicación. La comunicación OPC se realiza a través de una arquitectura cliente/servidor. 
Israel F. Benítez-Pina, Michel Sanz-Pérez, Jose Rogelio Martin-Escalona/ITEGAM-JETIA.Vol.01, No 04, pp.25-34. Dezembro, 2015.

El servidor OPC es la fuente de datos y cualquier aplicación basada en OPC puede acceder a dicho servidor para leer/escribir cualquier variable que ofrezca el servidor. Es una solución abierta y flexible al clásico problema de los drivers propietarios. Es por ello que prácticamente los mayores fabricantes de sistemas de control, instrumentación y de procesos han incluido OPC en sus productos.

El servidor OPC utilizado como enlace entre el sistema de supervisión y el autómata es un KEPware, el cual es un Servidor OPC DA, es decir, basado en la especificación de Acceso de Datos para la transmisión en tiempo real. La configuración de la comunicación del servidor OPC con el PLC se muestra en la figura 10. El SCADA y el PLC funcionarán como clientes del servidor OPC.

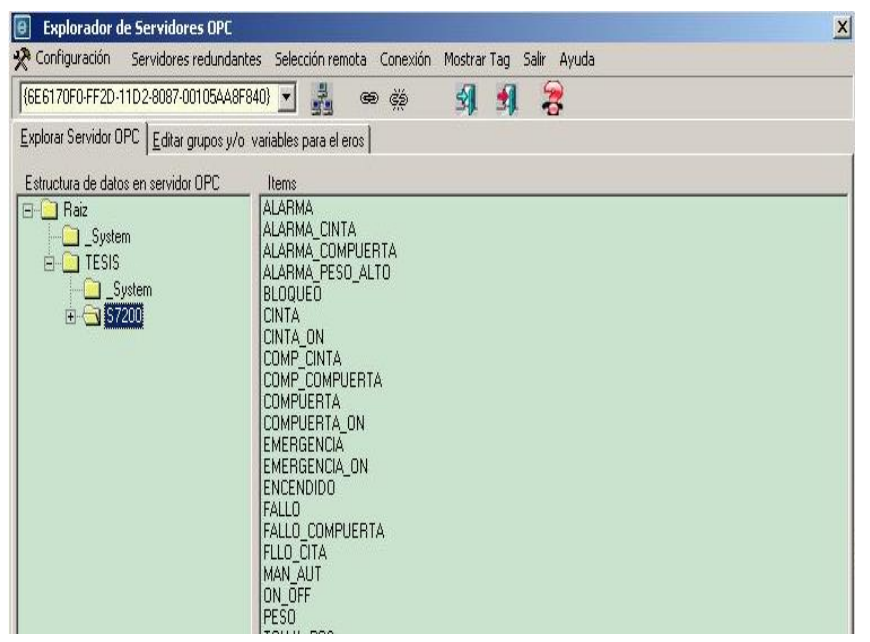

Figura 10 Enlace del EROS con el KEPware.

La configuración del servidor se puede verificar mediante el explorador del KEPware, donde se puede ver que las variables configuradas en el servidor están conectadas al simulador, coincidiendo los valores de las variables.

Luego de conformado todo el sistema se crearon diferentes guías de practicas de laboratorio para las diferentes asignaturas relacionadas con la instalación, así como sirve de plataforma de investigación científica para nuevas estrategias y algoritmos de control secuencial de la instalación. La misma ha sido utilizada con resultados satisfactorios y excelente acogida por los estudiantes de la carrera de automática de la Universidad de Oriente durante el curso pasado (2012-13) y en el actual. En la figura 11 se muestra la instalación en uso.

Con la puesta en marcha de la práctica de laboratorio los estudiantes cuentan con una herramienta capaz de acercarlos a un sistema de automatización real, lo que reafirma los conocimientos teóricos recibidos en clases y contribuye, de forma positiva, en su formación como ingenieros y futuros profesionales. De acuerdo a esto se logra consolidar los conocimientos adquiridos por los estudiantes en las asignaturas de Medios Técnicos de Automatización, Sistemas de Automatización, Autómatas Programables y cursos de postgrado, mediante diferentes prácticas de laboratorio.

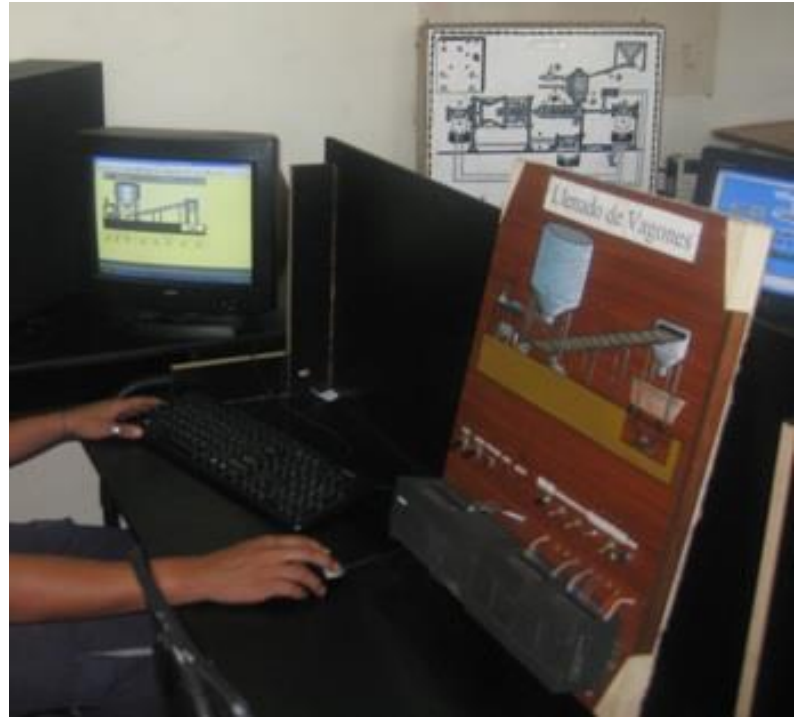

Figura 21. Uso de la instalación de laboratorio.

\section{CONSIDERACIONES FINALES}

Se implementó una maqueta del proceso de llenado de vagones y su sistema de automatización local y remota. Esta maqueta posibilita a los estudiantes consolidar los conocimientos adquiridos relacionados con los sistemas de automatización industrial.

Se implementó la programación de todo el sistema incluyendo el soporte para ejecución del supervisorio. A partir del modelo optimizado en Redes de Petri se pudo traducir el mismo al lenguaje de PLC. Se diseñó el sistema supervisorio del proceso empleando el sistema de supervisión y control EROS. Esto permite el entrenamiento de estudiantes de pre y postgrado en técnicas de diseño formal y programación de sistemas de automatización industrial y como plataforma de prueba para resultados de investigación científica en temas relacionados con esta aplicación.

Se agradece al grupo DesignLab del Dpto. de Mecatrónica de la EP-USP (Sao Paulo, Brasil) por el apoyo científico en la teoría de diseño formal mediante Redes de Petri GHENeSys (financiado por proyectos CAPES) y al CITCEA de la UPC (Barcelona, España) mediante la asistencia científicotécnica en automatización industrial con PLCs (financiado por proyectos CCD y ALFA).

\section{REFERÊNCIAS BIBLIOGRÁFICAS}

[1] Autómatas Industriales, Sistemas SCADA [en línea]. Marzo de 2006 [ref. Enero de 2012]. Disponible en Word Wide Web: <http://www.automatas.org/redes/scadas.htm>

[2] BAILEY, D; E. Wright, Practical SCADA for Industry. Editorial: Newnes (ELSEVIER), ISBN 07506 58053, 2003.

[3] BRENAN, R. Toward Real-Time Distributed Intelligent Control: A Survey of Research Themes and Applications. IEEE 
Israel F. Benítez-Pina, Michel Sanz-Pérez, Jose Rogelio Martin-Escalona/ITEGAM-JETIA.Vol.01, No 04, pp.25-34. Dezembro, 2015.

Trans. on Syst., Man, And Cyb. Part C, Vol. 37, No. 5, Issue Page(s): $744-765$. Sept. 2007.

[4] BENÍTEZ, I, Integración internacional de plataformas de enseñanza a distancia de automatización con PLC's, IEEERITA. Revista Iberoamericana de Tecnologías de Aprendizaje. Vol 1. No 1. Nov. ISSN 1932-8540, 2006.

[5] BENITEZ, I; Gomis, O; Sudriá, A; otros, Flexible manufacturing cell SCADA system for educational purposes. Journal Computer Applications in Engineering Education, Vol. 16, No 1, ISSN 1061-3773, Mayo 2008.

[6] COLOMBO, A. W., R. Schoop and R. Neubert (2006). An Agent-Based Intelligent Control Platform for Industrial Holonic Manufacturing Systems. IEEE Transactions on Industrial Electronics, Vol. 53, No. 1, PP. 322-337..

[7] DAVID R. and H. Alla, Discrete, Continuous and Hybrid Petri Nets. Berlin: Springer, 2004, (Revised 2nd edition, 2010).

[8] DRATH R. A mathematical approach to describing a class of hybrid systems. In: Proceedings of the IEEE Workshop on Parallel and Distributed Real Time Systems, Geneva, Switzerland, April 1997.

[9] FERRARINI, L., Dedè A., Salaün P., Duang, T., Fogliazza, G. Domain Specific Views in Model-driven Embedded Systems Design in Industrial Automation. 7th IEEE international Conference on Industrial Informatics.INDIN 2009.

[10] FREY, G.; Litz, L.: Formal methods in PLC programming. Proceedings of the IEEE SMC 2000, Nashville, TN, October 08-11, 2000.

[11] HAN, W. and M.A. Jafari. Component and Agent-Based FMS Modelling and Controller Synthesis. IEEE Transactions on Systems, Man and Cybernetics - Part C, Vol. 33, No. 2, pages 193-206. 2003

[12] LEITAO, P. Agent-based distributed manufacturing control: A state of the art survey. Eng. Applic. of Artif. Intell. Vol. 22, pp. 979-991. 2009.

[13] ESCALONA Martin, J.R. Sistema de Enseñanza de Automatización Integrada de Llenado de Vagones. Tesis de graduación en Automática. Dpto. Control Automático. Fac. de Ing. Eléctrica Univ. de Oriente. Santiago de Cuba. Junio 2012.

[14] MENDLING. J. Empirical Studies in Process Model Verification. K. Jensen and W. van der Aalst (Eds.): ToPNoC II, LNCS 5460, pp. 208-224, 2009. Springer-VerlagBerlin Heidelberg 2009.

[15] METZGER, M. \& Polaków, G. A Survey on Applications of Agent Technology in Industrial Process Control. IEEE Transactions on Industrial Informatics. Vol. 7, No. 4, November.
Pp570-581 2011.

[16] MIYAGI, P.E. Introdução a Simulação Discreta-Parte I. Departamento de Engenharia Mecatrônica e de Sistemas Mecânicos. Escola Politécnica da Universidade de São Paulo. Brasil. 2002.

[17] MURATA, Tadao (fellow IEEE. Department of Electrical Engineering and Computer Science, University of Illinois, Chicago). "Petri Nets: Properties, analysis and applications". Proceedings of IEEE, vol. 77, No. 4 April, 1989

[18] Object Management Group. Unified Modeling Language: Super structure Specification, version 2.1.2, August 2007. http://www.omg.org/spec/UML/2.1.2/Superstructure/PDF.

[19] VILLAJUlCA, J, ¿Qué es SCADA? [en línea]. 2011. http://www.instrumentacionycontrol.net/es/cursos-sistemasscada/146-que-es-scada-.html.

[20] SILVA J.R., Benítez I., Villafruela L., Gomis O., Sudriá A. "Modeling extended Petri nets compatible with GHENeSys IEC61131 for industrial automation". The International Journal of Advanced Manufacturing Technology. Springer London. Vol.36. Number 11-12 Pg. 1180-1190. UK. 2008. 